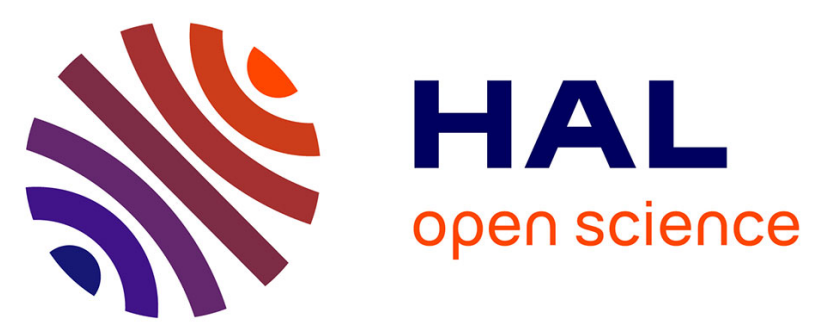

\title{
Channel Modeling and and Parameter Optimization for Hovering UAV-Based Free-Space Optical Links
}

Mohammad Taghi Dabiri, Seyed Mohammad Sajad Sadough, Mohammad Ali Khalighi

\section{- To cite this version:}

Mohammad Taghi Dabiri, Seyed Mohammad Sajad Sadough, Mohammad Ali Khalighi. Channel Modeling and and Parameter Optimization for Hovering UAV-Based Free-Space Optical Links. IEEE Journal on Selected Areas in Communications, 2018, Special issue on Airborne Communication Networks, 10.1109/JSAC.2018.2864416 . hal-02421604

\section{HAL Id: hal-02421604 https://hal.science/hal-02421604}

Submitted on 5 Apr 2020

HAL is a multi-disciplinary open access archive for the deposit and dissemination of scientific research documents, whether they are published or not. The documents may come from teaching and research institutions in France or abroad, or from public or private research centers.
L'archive ouverte pluridisciplinaire HAL, est destinée au dépôt et à la diffusion de documents scientifiques de niveau recherche, publiés ou non, émanant des établissements d'enseignement et de recherche français ou étrangers, des laboratoires publics ou privés. 


\title{
Channel Modeling and Parameter Optimization for Hovering UAV-Based Free-Space Optical Links
}

\author{
M.T. Dabiri, S.M.S. Sadough and M.A. Khalighi
}

\begin{abstract}
Recently, the use of multi-rotor (MR) unmanned aerial vehicles (UAVs) has emerged as a promising solution for establishing flexible free-space optical (FSO) communication links. We address in this paper accurate channel modeling to assess the benefits of MR UAV-based deployment for such links. In particular, in the absence of active tracking subsystems, we derive statistical models for ground-to-UAV, UAV-to-UAV, and UAV-to-ground links over both Gamma-Gamma and lognormal atmospheric turbulence models. Unlike previous works on this topic, our proposed model considers the joint effect of atmospheric turbulence along with position and angle-of-arrival fluctuations. The high accuracy of the proposed analytical models is verified by comparing numerically solved and Monte-Carlo simulation results in terms of link outage probability. We further discuss the impact of different transmitter/receiver parameters and their optimization in view of maximizing the link availability.
\end{abstract}

Index Terms-Free-space optics; unmanned aerial vehicles; angle-of-arrival fluctuations; atmospheric turbulence.

\section{INTRODUCTION}

D UE to the relatively high cost of deploying optical fibers, and the spectrum congestion that radio frequency systems are increasingly facing with, free-space optical (FSO) communications have recently attracted a growing attention as a cost-effective alternative technology for high data-rate point-to-point transmission in a wide range of applications [1] [3]. Nevertheless, the performance of FSO links is impaired by several factors including geometric and atmospheric loss, and atmospheric turbulence, which are all distance-dependent. Also, there is a stringent line-of-sight (LOS) requirement between the transmitter (Tx) and the receiver $(\mathrm{Rx})$ due to the typically narrow beams used [4]. Here, relay nodes can be inserted between the Tx and the Rx to improve link reliability an performance [5]. However, relay placement in optimal locations is not always possible, and particular attention should be devoted to the risk of exposure at rental relay locations. Recently, the use of unmanned aerial vehicles (UAV) has emerged as a promising solution [6]; but establishing such links of high reliability appeals for a number of challenges, including the necessity of precise link alignment [7].

Establishing aerial FSO links has been an important topic of research in the past. Initial works concerned laser communication for inter-satellite and satellite-to-ground links [1]. More recently, high-altitude platform (HAP)-based FSO networks

M. T. Dabiri and S. M. S. Sadough are with the Department of Electrical Engineering, Shahid Beheshti University G. C., 1983969411, Tehran (e-mail: s_sadough@sbu.ac.ir).

M. A. Khalighi is with the Aix Marseille University, CNRS, Centrale Marseille, Institut Fresnel, Marseille, France (e-mail: Ali.Khalighi@fresnel.fr). have received a great deal of attention [8]-[10]. Multi-rotor (MR) UAVs are an attractive option in relatively shorter links due to their advantages of high maneuverability, small size and low cost. They are increasingly employed in several applications, serving as mobile aerial base stations to enhance wireless connectivity [11]. There are, however, basic differences between satellite/HAP-based and MR UAV-based systems, especially, regarding the characteristics of the communication channel [12], which require dedicated study of the impact of different link parameters on the overall performance.

The literature on UAV-based FSO communications is relatively recent. In [6], experimental demonstration of a 100 m-roundtrip 80-Gbps orbital-angular-momentum-based FSO link was presented between a UAV and a ground station. In [13], the use of flying platforms was proposed for backhaul/fronthaul connectivity of cellular networks via FSO/milimeter-wave links. The benefits of deploying UAVs for relay-assisted FSO systems were investigated in [14]. A channel model was introduced in [15] for an FSO link between a UAV and a ground robot. Also, a tracking scheme for automatic alignment between mobile nodes was proposed in [16]. Open loop link alignment modeling was considered in [17] for an FSO link between two hovering UAVs.

To assess the benefits of UAV deployment for FSO communications, one important aspect is accurate channel modeling, which has been the subject of a few recent works. Most of these do not consider the effect of atmospheric turbulence that can severely affect the link performance. Another important factor is angle-of-arrival (AOA) fluctuations due to orientation deviations of hovering MR UAVs [12]. Indeed, for FSO links between fixed platforms, wind and thermal expansions result in random fluctuations in the aperture position and orientation, where the former parameter has the dominant effect [18]. For UAV-based links, however, the effect of AOA fluctuations should not be neglected. A recent work [19], considered channel modeling including the effects of AOA fluctuations, turbulence, and $\mathrm{Tx} / \mathrm{Rx}$ position fluctuations. There, to simplify the analytical derivations, the effect of Tx vibrations on AOA fluctuations was neglected by making far-field assumptions. Moreover, the results of [19] are obtained for log-normal turbulence whose validity is limited to the weak turbulence regime.

In this paper, assuming the absence of active tracking subsystems, we model a MR UAV-based FSO link in the presence of atmospheric turbulence by considering both position and AOA fluctuations. As illustrated in Fig. 1, a general MR UAVbased link can be divided into three different links: groundto-UAV (GU), UAV-to-UAV (UU) and UAV-to-ground (UG). 


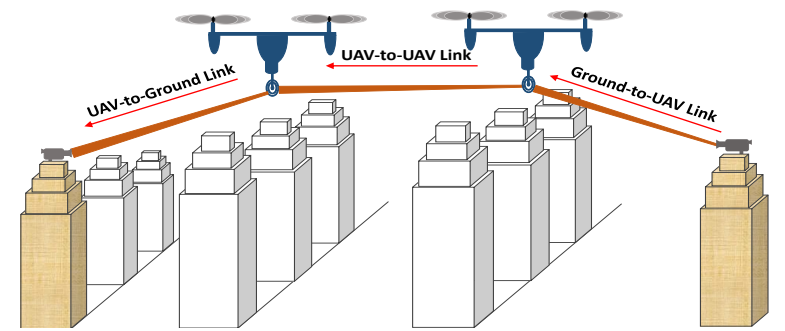

Fig. 1. Illustration of a typical MR UAV-based FSO communication link.

Our performance evaluation is mainly based on the outage probability, which is the most relevant metric for quasi-static FSO channels. As we will show later, each channel depends on nine random variables (RVs). We provide closed-form expressions for the probability density function (PDF) of the three link types, as well as analytical expressions for the outage probability over both Gamma-Gamma (GG) and lognormal (LN) atmospheric turbulence models, which require only one-dimensional integration. Moreover, we provide a detailed and insightful analysis of the parameter optimization of the Tx/Rx such as the Tx beam-width and the Rx field-ofview (FOV) that can be quite useful for the practical design of these links. In Section II, we describe our system model and main assumptions. Analytical formulations of the channel PDF for the MR UAV-based links are developed then in Section III. Next, Section IV presents numerical results to show the accuracy of the proposed models as well as to study the link performance and parameter optimization. Lastly, in Section V, we present our main conclusions and a few future research directions.

\section{System Model And Main Assumptions}

Figure 2 illustrates the 2-D schematic diagram of a UU system consisting of two hovering UAVs. To model the UU link parameters in 3-D space, we make the following assumptions:

- the two highly stable aerial nodes hover in one place;

- in the Cartesian coordinate system $[x, y, z] \in \mathbb{R}^{1 \times 3}$, the mean positions of the Tx and Rx are at $A_{T_{x}}=[0,0,0]$ and $A_{R_{x}}=[0,0, Z]$, respectively, and are perfectly known to $\mathrm{Tx} / \mathrm{Rx}$, which can be realized through periodic data exchange between them;

- the mean orientation vectors of the Tx and the Rx are located on the $z$ axis;

- instantaneous position and orientation of the Tx and the Rx are slightly deviated from their means under the effect of numerous random events related to UAV hovering;

- no spatial or temporal tracking is done.

Within the Cartesian coordinates, RVs $A_{T_{x}}^{\prime}=\left[x_{t}, y_{t}, z_{t}\right]$ and $A_{R_{x}}^{\prime}=\left[x_{r}, y_{r}, Z+z_{r}\right]$ denote the instantaneous positions of the Tx and the Rx nodes, respectively, with respect to their mean $A_{T_{x}}^{\prime}$ and $A_{R_{x}}^{\prime}$. We assume that $x_{t}, x_{r}, y_{t}, y_{r}, z_{t}$ and $z_{r}$ are very small, compared with the link range $Z$. Then, angles $\varphi_{t x}$, $\varphi_{t y}, \varphi_{r x}$ and $\varphi_{r y}$, indicated in Fig. 2, can be approximated as: $\varphi_{t x} \simeq \frac{x_{t}}{Z}, \varphi_{t y} \simeq \frac{y_{t}}{Z}, \varphi_{r x} \simeq \frac{x_{r}}{Z}$ and $\varphi_{r y} \simeq \frac{y_{r}}{Z}$.

In addition to the random displacements, the optical axes of the Tx and Rx also vary randomly. In $[x, z]$ and $[y, z]$ Cartesian coordinates, we denote the instantaneous Tx and Rx misalignment orientations by $\theta_{t x}, \theta_{t y}, \theta_{r x}$ and $\theta_{r y}$, respectively.

We consider highly stable hovering MR UAVs which offer high angular stability on the order of mrad thanks to the accuracy of mechanical and control systems. Thus, assuming that $\theta_{t x}$ and $\theta_{t y}$ are sufficiently small, we can use small-angle approximation as follows.

$$
\begin{aligned}
& x_{\theta_{t x}}=\tan \left(\theta_{t x}\right) Z \simeq \theta_{t x} Z, \\
& y_{\theta_{t y}}=\tan \left(\theta_{t y}\right) Z \simeq \theta_{t y} Z,
\end{aligned}
$$

where $x_{\theta_{t x}}$ and $y_{\theta_{t y}}$ denote the beam position deviations at the Rx, respectively. Based on the central limit theorem, position and orientation deviations are considered as Gaussian distributed as they result from numerous random events [12]. We assume that the variances of position deviations are the same in $x, y$ and $z$ axes. Therefore, considering the same conditions for the two hovering MR UAVs, $x_{t}, x_{r}, y_{t}, y_{r}, z_{t}$ and $z_{r}$ are modeled as zero-mean independent Gaussian RVs with variance $\sigma_{p}^{2}$, and $\theta_{t x}, \theta_{t y}, \theta_{r x}$ and $\theta_{r y}$ are modeled as zero-mean independent Gaussian RVs with variance $\sigma_{\theta}^{2}$.

\section{A. Received Signal Model}

Typically a converging lens is used before the photo-detector (PD), which converts the received intensity to a photo-current. The $\mathrm{Rx}$ also collects undesired background radiations due mainly to scattered sunlight [20], [21]. Subtracting a constant bias due to the background noise mean power, the PD output corresponding to the $k$ th symbol interval, i.e., $\left[(k-1) T_{b}, k T_{b}\right)$ with $T_{b}$ being the symbol duration, can be written as [22]

$$
r[k]=R h s[k]+n[k],
$$

where $h$ denotes the channel attenuation coefficient, $n[k]$ is the photo-current noise, $s[k]$ is the transmitted symbol with average optical power $P_{t}$, and $R$ is the PD responsitivity. We assume that the background noise is the dominant noise source at the Rx and consider $n[k]$ as a signal-independent zero-mean Gaussian noise with variance $\sigma_{n}^{2}=2 e B_{e} R P_{b}$, where $e$ denotes the electron charge and $B_{e}$ is the PD bandwidth (in $\mathrm{Hz}$ ). Furthermore, $P_{b}$ denotes the background power, which can be formulated as $P_{b}=N_{b}(\lambda) B_{o} \Omega_{F O V} A_{a}$, where $N_{b}(\lambda)$ is the spectral radiance of the background radiations at wavelength $\lambda$ (in Watts $/ \mathrm{cm}^{2}-\mu \mathrm{m}$-srad), $B_{o}$ is the bandwidth of the optical filter at the $\mathrm{Rx}$ (in $\mu \mathrm{m}$ ) and $A_{a}$ is the lens area $\left(\right.$ in $\mathrm{cm}^{2}$ ) [23, Eq. (3b)]. Moreover, $\Omega_{F O V}$ denotes the Rx's FOV (in srad) that can be obtained as $\Omega_{F O V}=2 \pi\left(1-\cos \left(\theta_{F O V} / 2\right)\right) \simeq$ $\pi \theta_{F O V}^{2} / 4$. The instantaneous electrical signal-to-noise ratio (SNR) is defined as $\Upsilon=R^{2} P_{t}^{2} h^{2} / \sigma_{n}^{2}$. In slow fading channels, which is typical of FSO links, the outage probability $\mathcal{P}_{\text {out }}$ (i.e., the probability that the instantaneous SNR falls below a threshold $\Upsilon_{t h}$ ) is the most appropriate performance metric. We define $\mathcal{P}_{\text {out }}$ as

$$
\mathcal{P}_{\text {out }}=\int_{0}^{\Upsilon_{t h}} f_{\Upsilon}(\Upsilon) d \Upsilon=\int_{0}^{h_{t h}} f_{h}(h) d h,
$$

where

$$
h_{t h}=\sqrt{\sigma_{n}^{2} \Upsilon_{t h}} / R P_{t},
$$

and $f_{\Upsilon}$ and $f_{h}$ denote the PDFs of $\Upsilon$ and $h$, respectively. 


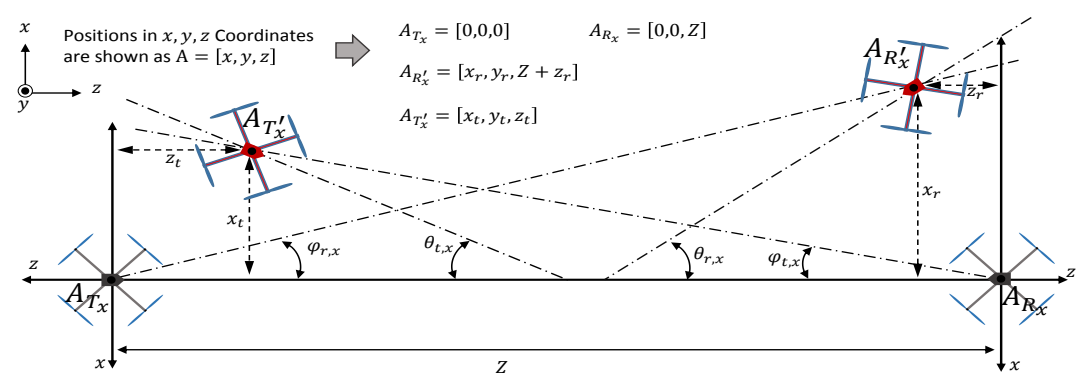

Fig. 2. Schematic 2-D diagram of a UU system consisting of two hovering MR UAV nodes. $A_{T_{x}}$ and $A_{R_{x}}$ are the mean of Tx and Rx positions, and $A_{T_{x}}^{\prime}$ and $A_{R_{x}}^{\prime}$ show the deviations from $A_{T_{x}}$ and $A_{R_{x}}$, respectively.

\section{Channel Modeling}

The considered source-to-destination link involves three different channels, i.e., GU, UU, and UG, see Fig. 1. We first develop the channel model for the UU link, based on which we later provide the model for the GU and UG channels.

\section{A. UAV-to-UAV Channel Modeling}

Our model takes into account four impairments, i.e., the atmospheric attenuation $h_{l}$, the atmospheric turbulence $h_{a}$, the pointing error loss $h_{p g}$, and the link interruption $h_{p a}$ due to the AOA fluctuations. The UU channel coefficient is then,

$$
h=h_{l} h_{a} h_{p g} h_{p a} .
$$

1) Atmospheric Attenuation and Turbulence: The atmospheric attenuation is typically modeled by the Beer-Lambert law as $h_{l}=\exp (-Z \xi)$, with $\xi$ being the scattering coefficient, which is a function of visibility [2]. Considering the atmospheric turbulence induced fading, we use LN and GG models; the former is appropriate for weak turbulence conditions whereas the latter can describe all turbulence regimes [2], [24]. The distribution of $h_{a}$ according to the $\mathrm{LN}$ model is

$$
f_{\mathrm{L}}\left(h_{a}\right)=\frac{1}{2 h_{a} \sigma_{\operatorname{Ln} h_{a}} \sqrt{2 \pi}} \exp \left(-\frac{\left(\ln \left(h_{a}\right)-2 \mu_{\operatorname{Ln} h_{a}}\right)^{2}}{8 \sigma_{\operatorname{Ln} h_{a}}^{2}}\right) .
$$

where $\mu_{\mathrm{Lnh} h_{a}}$ and $\sigma_{\mathrm{Lnh} h_{a}}^{2}$ denote the mean and variance of log-irradiance, respectively, where $\sigma_{\mathrm{Ln} h_{a}}^{2} \simeq \sigma_{R}^{2} / 4$ with $\sigma_{R}^{2}$ being the Rytov variance [25]. Setting $\mathbb{E}\left[h_{a}\right]=1$ (with $\mathbb{E}[$. denoting the expected value) we have $\mu_{\operatorname{Ln} h_{a}}=-\sigma_{\operatorname{Ln} h_{a}}^{2}$.

For the GG model, the distribution of the random variable $h_{a}$ is given by [24]

$$
f_{\mathrm{G}}\left(h_{a}\right)=\frac{2(\alpha \beta)^{\frac{\alpha+\beta}{2}}}{\Gamma(\alpha) \Gamma(\beta)} h_{a}^{\frac{\alpha+\beta}{2}-1} k_{\alpha-\beta}\left(2 \sqrt{\alpha \beta h_{a}}\right),
$$

where $\Gamma($.$) is the Gamma function and k_{n}($.$) is the modified$ Bessel function of the second kind of order $n$. Also, $\alpha$ and $\beta$ are respectively the effective number of large-scale and smallscale eddies, which depend on Rytov variance $\sigma_{R}^{2}$ [24]. Unless otherwise mentioned, we consider the GG channel model in the following.

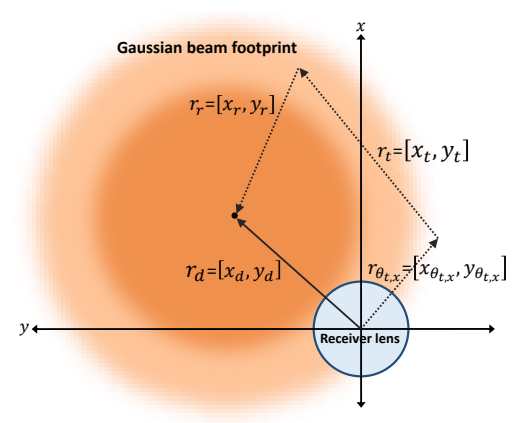

Fig. 3. Gaussian beam footprint at the Rx aperture. The center of received beam is deviated from the center of $\mathrm{Rx}$ lens due to the random displacement of hovering Tx and Rx and also due to the orientation deviation of Tx.

2) Pointing Error Loss: We consider a Gaussian beam at the Tx, for which the normalized spatial distribution of the transmitted intensity at distance $Z$, is given by [24]

$$
I_{r}(r, Z)=\frac{2}{\pi w_{z}} \exp \left(-\frac{2\left(x^{2}+y^{2}\right)}{w_{z}^{2}}\right),
$$

where $r=[x, y]$ is the radial distance vector from the beam center and $w_{z}$ is the beam waist at distance $Z$. Let us consider the beam spot as shifted to the position $r_{d}=\left[x_{d}, y_{d}\right]$ with respect to the center of the Rx lens, as depicted in Fig. 3. The RVs $x_{d}$ and $y_{d}$ are obtained as

$$
x_{d}=x_{t r}+Z \theta_{t x}, \text { and } y_{d}=y_{t r}+Z \theta_{t y},
$$

where $x_{t r}=x_{t}+x_{r}$ and $y_{t r}=y_{t}+y_{r}$ are zero mean Gaussian RVs with variance $2 \sigma_{p}^{2}$. Considering the Rx lens radius $r_{a}$, the pointing error loss $h_{p g}$ (which includes the geometric loss as well) can be expressed as

$$
\begin{aligned}
& h_{p g}=\int_{-r_{a}}^{r_{a}} \int_{-\sqrt{r_{a}^{2}-y^{2}}}^{\sqrt{r_{a}^{2}-y^{2}}} \frac{2}{\pi w_{z}} \\
& \times \exp \left(-2 \frac{\left(x+x_{d}\right)^{2}+\left(y+y_{d}\right)^{2}}{w_{z}^{2}}\right) d x d y .
\end{aligned}
$$

Using [18, Eqs. (8), (9)], (10) can be approximated as

$$
h_{p g} \simeq A_{0} \exp \left(-2 r_{t r}^{2} / w_{z_{\mathrm{eq}}}^{2}\right),
$$

where $r_{t r}=\sqrt{x_{d}^{2}+y_{d}^{2}}$. The parameter $A_{0}=(\operatorname{erf}(\nu))^{2}$ denotes the maximal fraction of the collected intensity with 
$\nu=\frac{\sqrt{\pi} r_{a}}{\sqrt{2} w_{z}}, w_{z_{\mathrm{eq}}}^{2}=w_{z}^{2} \frac{\sqrt{\pi} \operatorname{erf}(\nu)}{2 \nu \exp \left(-\nu^{2}\right)}$ is the equivalent beam waist, and $\operatorname{erf}($.$) is the error function. Since the RVs x_{d}$ and $y_{d}$ conditioned on $\theta_{t x}$ and $\theta_{t y}$, respectively, have a Gaussian distribution as $x_{d} \sim \mathcal{N}\left(\theta_{t x} Z, 2 \sigma_{p}^{2}\right)$ and $y_{d} \sim \mathcal{N}\left(\theta_{t y} Z, 2 \sigma_{p}^{2}\right)$, $r_{t r}$ conditioned on $\theta_{t x}$ and $\theta_{t y}$ has a Rician distribution as

$$
f_{r_{t r} \mid \theta_{x y}}\left(r_{t r}\right)=\frac{r_{t r}}{2 \sigma_{p}^{2}} \exp \left(-\frac{r_{t r}^{2}+\theta_{x y}^{2} Z^{2}}{4 \sigma_{p}^{2}}\right) I_{0}\left(\frac{Z \theta_{x y} r_{t r}}{2 \sigma_{p}^{2}}\right) .
$$

$I_{0}($.$) is the modified Bessel function of the first kind with$ order zero, and $\theta_{x y}=\sqrt{\theta_{t x}^{2}+\theta_{t y}^{2}}$ is Rayleigh distributed as:

$$
f_{\theta_{x y}}\left(\theta_{x y}\right)=\left(\theta_{x y} / \sigma_{\theta}^{2}\right) \times \exp \left(-\theta_{x y}^{2} / 2 \sigma_{\theta}^{2}\right) .
$$

According to (11) and (12) and after some algebra, the PDF of $h_{p g}$ conditioned on $\theta_{x y}$ is derived as

$$
\begin{aligned}
f_{h_{p g} \mid \theta_{x y}}\left(h_{p g}\right)= & \frac{w_{z_{e q}}^{2}}{4 h_{p g} \sqrt{-\frac{w_{z_{e q}}^{2}}{2} \ln \left(\frac{h_{p g}}{A_{0}}\right)}} \\
& \times f_{r_{t r} \mid \theta_{x y}}\left(\sqrt{\left.-\frac{w_{z_{e q}}^{2}}{2} \ln \left(\frac{h_{p g}}{A_{0}}\right)\right)}\right) \\
= & \frac{w_{z_{e q}}^{2}}{8 A_{0} \sigma_{p}^{2}} \exp \left(-\frac{\theta_{x y}^{2} Z^{2}}{4 \sigma_{p}^{2}}\right)\left(\frac{h_{p g}}{A_{0}}\right) \frac{w_{z_{e q}}^{2}}{8 \sigma_{p}^{2}}-1 \\
& \times I_{0}\left(\frac{Z \theta_{x y}}{2 \sigma_{p}^{2}} \sqrt{-\frac{w_{z_{e q}}^{2}}{2} \ln \left(\frac{h_{p g}}{A_{0}}\right)}\right) \\
& \text { for } 0 \leq h_{p g} \leq A_{0}
\end{aligned}
$$

Considering the GG turbulence model, from (7), (13) and (14), the distribution of $h^{\prime}=h_{l} h_{a} h_{p g}$ conditioned on $\theta_{x y}$ is obtained as

$$
\begin{aligned}
f_{h^{\prime} \mid \theta_{x y}}^{\mathrm{G}}\left(h^{\prime}\right)= & \int_{0}^{\infty} f_{h^{\prime} \mid h_{a}, \theta_{x y}}\left(h^{\prime}\right) f_{h_{a}}\left(h_{a}\right) d h_{a} \\
= & \int_{0}^{\infty} \frac{1}{h_{a} h_{l}} f_{h_{p g} \mid \theta_{x y}}\left(\frac{h^{\prime}}{h_{a} h_{l}}\right) f_{h_{a}}\left(h_{a}\right) d h_{a} \quad \text { (15) } \\
= & \frac{2(\alpha \beta)^{\frac{\alpha+\beta}{2}}}{\Gamma(\alpha) \Gamma(\beta)} \frac{\gamma_{u u}^{2} h^{\prime \gamma_{u u}^{2}-1}}{\left(A_{0} h_{l}\right)^{\gamma_{u u}^{2}}} \exp \left(-\frac{\theta_{x y}^{2} Z^{2}}{4 \sigma_{p}^{2}}\right) \\
& \times \int_{\frac{h^{\prime}}{A_{0} h_{l}}}^{\infty} I_{0}\left(\frac{Z \theta_{x y}}{2 \sigma_{p}^{2}} \sqrt{-\frac{w_{z_{e q}}^{2}}{2} \ln \left(\frac{h^{\prime}}{A_{0} h_{l} h_{a}}\right)}\right) \\
& \times h_{a}^{\frac{\alpha+\beta}{2}-\gamma_{u u}-1} k_{\alpha-\beta}\left(2 \sqrt{\alpha \beta h_{a}}\right) d h_{a} .
\end{aligned}
$$

where $\gamma_{u u}^{2}=w_{z_{e q}}^{2} / 8 \sigma_{p}^{2}$. Using [26, Eq. (03.04.06.0002.01)], the modified Bessel function of the second kind $k_{\nu}(x)$ can be approximated as

$$
k_{\nu}(x)=\frac{\pi}{2 \sin (\pi \nu)} \sum_{n=0}^{\infty}\left[\frac{(x / 2)^{2 n-\nu}}{\Gamma(n-\nu+1) n !}-\frac{(x / 2)^{2 n+\nu}}{\Gamma(n+\nu+1) n !}\right] .
$$

Applying a change of variable $z=\sqrt{-\frac{w_{z_{e q}}^{2}}{2} \ln \left(\frac{h^{\prime}}{A_{0} h_{l} h_{a}}\right)}$ and using (17), (62), [27, Eqs. (6.643.2) and (9.227)], after some manipulations, a closed form expressions for (16) is derived as

$$
\begin{aligned}
f_{h^{\prime} \mid \theta_{x y}}^{\mathrm{G}}\left(h^{\prime}\right) \simeq & \sum_{j=0}^{J}\left(\mathcal{A}_{j}(\alpha, \beta) e^{-a_{j} \theta_{x y}^{2}} h^{\prime \beta-1+j}\right. \\
& \left.-\mathcal{A}_{j}(\beta, \alpha) e^{-b_{j} \theta_{x y}^{2}} h^{\prime \alpha-1+j}\right),
\end{aligned}
$$

where

$$
\mathcal{A}_{j}(\alpha, \beta)=\frac{\pi \gamma_{u u}^{2}\left(\frac{\alpha \beta}{A_{0} h_{l}}\right)^{\beta} \sin ^{-1}((\alpha-\beta) \pi)}{\Gamma(\alpha) \Gamma(\beta) \Gamma(-(\alpha-\beta)+1)\left|-\left(\beta-\gamma_{u u}^{2}\right)\right|},
$$

and $a_{j}=\frac{Z^{2}}{4 \sigma_{p}^{2}}+\frac{Z^{2} \gamma_{u u}^{2}}{4 \sigma_{p}^{2}\left(\beta-\gamma_{u u}^{2}+j\right)}, b_{j}=\frac{Z^{2}}{4 \sigma_{p}^{2}}+\frac{Z^{2} \gamma_{u u}^{2}}{4 \sigma_{p}^{2}\left(\alpha-\gamma_{u u}^{2}+j\right)}$ and $J=\left\lfloor\gamma_{u u}^{2}-\alpha\right\rfloor$.

3) Link Interruption due to AOA Fluctuations: Let us define the AOA of the signal as the incidence angle relative to the $\mathrm{Rx}$ axis that we denote by $\theta_{a}$. According to (1), $\theta_{a}$ can be closely approximated as

$$
\theta_{a} \simeq \sqrt{\left(\theta_{t x}+\theta_{r x}\right)^{2}+\left(\theta_{t y}+\theta_{r y}\right)^{2}}
$$

Given the limited Rx FOV, a link interruption occurs for $\theta_{a}>\theta_{F O V}$. Thus the corresponding loss $h_{p a}$ due to AOA fluctuations can be written as

$$
h_{p a}=\Pi\left(\theta_{a} / \theta_{F O V}\right),
$$

where $\Pi(x)=1$ if $|x| \leq 1$, and $\Pi(x)=0$ otherwise.

4) Overall Channel Model: According to link interruption formulation in (19), (20) and after some derivations provided in Appendix A, we obtain the distribution of $h$ conditioned on $\theta_{x y}$ as

$$
\begin{aligned}
f_{h \mid \theta_{x y}}(h)= & F_{\theta_{a} \mid \theta_{x y}}\left(\theta_{F O V}\right) f_{h^{\prime} \mid \theta_{x y}}(h) \\
& +\left(1-F_{\theta_{a} \mid \theta_{x y}}\left(\theta_{F O V}\right)\right) \delta(h),
\end{aligned}
$$

where

$$
F_{\theta_{a} \mid \theta_{x y}}\left(\theta_{F O V}\right) \simeq 1-\exp \left(-\frac{\theta_{x y}^{2}}{2 \sigma_{\theta}^{2}}\right) \sum_{m=0}^{M} \mathcal{H}(m)\left(\frac{\theta_{x y}^{2}}{\sigma_{\theta}^{2}}\right)^{m},
$$

and $\delta($.$) is Dirac delta function [27] and \mathcal{H}(m)$ is defined in (46). Lastly, the PDF of $h$ can be obtained as

$$
f_{h}(h)=\int_{0}^{\infty} f_{h \mid \theta_{x y}}(h) f_{\theta_{x y}}\left(\theta_{x y}\right) d \theta_{x y}
$$

Substituting (13), (18), (21) and (22) in (23), we obtain the PDF of $h$ for UU link as

$$
f_{G}^{u u}(h)=f_{G}^{u u}(h>0)+f_{G}^{u u}(h=0) \delta(h),
$$

where $f_{G}^{u u}(h>0)$ is expressed as

$$
\begin{aligned}
& f_{G}^{u u}(h>0) \simeq \\
& \sum_{j=0}^{J} \int_{0}^{\infty} \frac{\theta_{x y}}{\sigma_{\theta}^{2}}\left(1-\exp \left(-\frac{\theta_{x y}^{2}}{2 \sigma_{\theta}^{2}}\right) \sum_{m=0}^{M} \mathcal{H}(m)\left(\frac{\theta_{x y}^{2}}{\sigma_{\theta}^{2}}\right)^{m}\right) \\
& \times\left(\mathcal{A}_{j}(\alpha, \beta) \exp \left(-\left(a_{j}+\frac{1}{2 \sigma_{\theta}^{2}}\right) \theta_{x y}^{2}\right) h^{\beta-1+j}\right. \\
& \left.-\mathcal{A}_{j}(\beta, \alpha) \exp \left(-\left(b_{j}+\frac{1}{2 \sigma_{\theta}^{2}}\right) \theta_{x y}^{2}\right) h^{\alpha-1+j}\right) d \theta_{x y} .
\end{aligned}
$$


Also, by using [27, Eq. (3.461.3)], a closed-form expression for $f_{G}^{u u}(h=0)$ can be derived as

$$
\begin{aligned}
f_{G}^{u u}(h=0) & =\int_{0}^{\infty} \frac{\theta_{x y}}{\sigma_{\theta}^{2}} \exp \left(-\frac{\theta_{x y}^{2}}{\sigma_{\theta}^{2}}\right) \sum_{m=0}^{M} \mathcal{H}(m)\left(\frac{\theta_{x y}^{2}}{\sigma_{\theta}^{2}}\right)^{m} d \theta_{x y} \\
& =\sum_{m=0}^{M} \frac{\mathcal{H}(m) m !}{2} .
\end{aligned}
$$

Using [27, Eq. (3.351.3)] and after some mathematical derivations, the closed form expression for (25) is derived as

$$
\begin{aligned}
f_{G}^{u u}(h>0) \simeq & \sum_{j=0}^{J}\left(\frac{\mathcal{A}_{j}(\alpha, \beta)}{1+2 a_{j} \sigma_{\theta}^{2}} h^{\beta-1+j}-\frac{\mathcal{A}_{j}(\beta, \alpha,)}{1+2 b_{j} \sigma_{\theta}^{2}} h^{\alpha-1+j}\right) \\
& -\sum_{m=0}^{M} \sum_{j=0}^{J} \frac{\mathcal{H}(m)}{2 \sigma_{\theta}^{2 m+2}}\left(\frac{m ! \mathcal{A}_{j}(\alpha, \beta)}{\left(1+a_{j} \sigma_{\theta}^{2}\right)^{m+1}} h^{\beta-1+j}\right. \\
& \left.-\frac{m ! \mathcal{A}_{j}(\beta, \alpha)}{\left(1+b_{j} \sigma_{\theta}^{2}\right)^{m+1}} h^{\alpha-1+j}\right) .
\end{aligned}
$$

For low values of $h$, (27) can be simplified as

$$
\begin{aligned}
& f_{G}^{u u}(h>0) \simeq \frac{\pi \gamma_{u u}^{2}\left(\frac{\alpha \beta}{A_{0} h_{l}}\right)^{\beta} \sin ^{-1}((\alpha-\beta) \pi)}{\Gamma(\alpha) \Gamma(\beta) \Gamma(-(\alpha-\beta)+1)\left|-\left(\beta-\gamma_{u u}^{2}\right)\right|} \\
& \times\left(\frac{1}{1+2 a_{0} \sigma_{\theta}^{2}}-\frac{1}{2} \sum_{m=0}^{M} \frac{m ! \mathcal{H}(m)}{\left(1+a_{0} \sigma_{\theta}^{2}\right)^{m+1}}\right) h^{\beta-1} \cdot
\end{aligned}
$$

Note that, as we will explain later, (28) is valid for relatively low values of $h$, which means that it suitable for performance analysis at relatively low $\mathcal{P}_{\text {out }}$ or low bit-error-rate.

For the sake of completeness, we have provided in Appendix $\mathrm{B}$ the PDF of $\mathrm{h}$ for UU link under LN turbulence model, see (49). Also, we have considered in Appendix C the special case where the turbulence effect is negligible; the channel distribution is then given by (58).

\section{B. Ground-to-UAV Channel Model}

For the GU link, we take into consideration the deviations in the position and the orientation of the Tx due to building sway, i.e., the RVs $x_{t}, y_{t}, \theta_{t x}$, and $\theta_{t y}$ [18]. For a typical Tx mounted on a building, $\theta_{t x}$ and $\theta_{t y}$ are usually very small and can be set to zero [4]. As a result, we consider $\theta_{x y} \simeq 0$. Also, we assume that the variances of Tx position fluctuations in $x$ and $y$ axes are the same and equal to $\sigma_{g}^{2}$. Then, (12) can be simplified to the Rayleigh distribution, as follows:

$$
f_{r_{t r}}\left(r_{t r}\right)=\left(r_{t r} / \sigma_{p g}^{2}\right) \exp \left(-r_{t r}^{2} / 2 \sigma_{p g}^{2}\right)
$$

where $\sigma_{p g}^{2}=\sigma_{p}^{2}+\sigma_{g}^{2}$. According to (7), (29) and (15), the distribution of $h^{\prime}$ for the GU link is obtained as

$$
\begin{aligned}
f_{h^{\prime}}^{\mathrm{G}}\left(h^{\prime}\right) & =\frac{2 \gamma_{g u}^{2}(\alpha \beta)^{\frac{\alpha+\beta}{2}}}{\Gamma(\alpha) \Gamma(\beta)\left(A_{0} h_{l}\right)^{\gamma_{g u}^{2}}} h^{\prime \gamma_{g u}^{2}-1} \\
& \times \int_{h^{\prime} / A_{0} h_{l}}^{\infty} h_{a}^{\frac{\alpha+\beta}{2}-1-\gamma_{g u}^{2}} k_{\alpha-\beta}\left(2 \sqrt{\alpha \beta h_{a}}\right) d h_{a} .
\end{aligned}
$$

where $\gamma_{g u}^{2}=\frac{w_{z_{e q}}^{2}}{4 \sigma_{p g}^{2}}$. Similar to the derivation of (18), the closed form expression for (30) is obtained as

$$
f_{h^{\prime}}^{\mathrm{G}}\left(h^{\prime}\right) \simeq \sum_{j=0}^{J^{\prime}}\left(\mathcal{A}_{j}^{g u}(\alpha, \beta) h^{\prime \beta-1+j}-\mathcal{A}_{j}^{g u}(\beta, \alpha) h^{\alpha-1+j}\right),
$$

where $J^{\prime}=\left\lfloor\gamma_{g u}^{2}-\alpha\right\rfloor$ and $\mathcal{A}_{j}^{g u}(\alpha, \beta)$ is obtained from $\mathcal{A}_{j}(\alpha, \beta)$ by substituting $\gamma_{g u}$ instead of $\gamma_{u u}$.

For GU link, $\theta_{a}=\sqrt{\theta_{r x}^{2}+\theta_{r y}^{2}}$, which has a Rayleigh distribution as

$$
f_{\theta_{a}}\left(\theta_{a}\right)=\left(\theta_{a} / \sigma_{\theta}^{2}\right) \times \exp \left(-\theta_{a}^{2} / 2 \sigma_{\theta}^{2}\right) .
$$

According to (43) and (32), the distribution of $h_{p a}$ can now be derived as follows

$$
\begin{aligned}
f_{h_{p a}}\left(h_{p a}\right)= & {\left[1-\exp \left(-\theta_{F O V}^{2} / 2 \sigma_{\theta}^{2}\right)\right] \delta\left(h_{p a}-1\right) } \\
& +\exp \left(-\theta_{F O V}^{2} / 2 \sigma_{\theta}^{2}\right) \delta\left(h_{p a}\right) .
\end{aligned}
$$

Then, from (21), (31) and (33), the distribution of $h$ for GU link can be obtained as

$$
f_{G}^{g u}(h)=f_{G}^{g u}(h>0)+f_{G}^{g u}(h=0) \delta(h),
$$

where

$$
f_{G}^{g u}(h=0)=\int_{0}^{\theta_{F O V}} f_{\theta_{a}}\left(\theta_{a}\right) d \theta_{a}=\exp \left(-\theta_{F O V}^{2} / 2 \sigma_{\theta}^{2}\right),
$$

and $f_{G}^{g u}(h>0)$ is obtained as

$$
\begin{aligned}
& f_{G}^{g u}(h>0)=\left[1-\exp \left(-\theta_{F O V}^{2} / 2 \sigma_{\theta}^{2}\right)\right] \\
& \quad \times \sum_{j=0}^{J^{\prime}}\left(\mathcal{A}_{j}^{g u}(\alpha, \beta) h^{\prime \beta-1+j}-\mathcal{A}_{j}^{g u}(\beta, \alpha) h^{\prime \alpha-1+j}\right) .
\end{aligned}
$$

For low values of $h,(36)$ can be simplified as

$$
\begin{aligned}
& f_{G}^{g u}(h>0)=\left[1-\exp \left(-\theta_{F O V}^{2} / 2 \sigma_{\theta}^{2}\right)\right] \\
& \quad \times \frac{\pi \gamma_{g u}^{2}\left(\frac{\alpha \beta}{A_{0} h_{l}}\right)^{\beta} \sin ^{-1}((\alpha-\beta) \pi) h^{\beta-1}}{\Gamma(\alpha) \Gamma(\beta) \Gamma(-(\alpha-\beta)+1)\left|-\left(\beta-\gamma_{g u}^{2}\right)\right|} .
\end{aligned}
$$

The PDF of $h$ for GU link under LN turbulence is provided in Appendix B, see (53). Moreover, in Appendix C, we have presented the GU link channel model for the case of negligible turbulence.

\section{UAV-to-Ground Channel Model}

For the UG link, the $\mathrm{Rx}$ is fixed and we set $\theta_{r x}$ and $\theta_{r y}$ to zero, resulting in $\theta_{a}=\theta_{x y}$. Consequently, we have $F_{\theta_{a} \mid \theta_{x y}}\left(\theta_{F O V}\right)=F_{\theta_{a}}\left(\theta_{F O V}\right)=\Pi\left(\frac{\theta_{x y}}{\theta_{F O V}}\right)$. Following (12)(48), $f_{h^{\prime} \mid \theta_{x y}}$ for the UG link is similar to the case of the UU link when we replace $\sigma_{p g}^{2}$ by $2 \sigma_{p}^{2}$ in (48). Based on (21), $f_{h \mid \theta_{x y}}$ is derived as follows.

$$
f_{h \mid \theta_{x y}}(h)=f_{h^{\prime} \mid \theta_{x y}}(h) \Pi\left(\frac{\theta_{x y}}{\theta_{F O V}}\right)+\left[1-\Pi\left(\frac{\theta_{x y}}{\theta_{F O V}}\right)\right] \delta(h) .
$$

Then, using (13), (18), (38) and [27, Eq. (3.323.3)], the PDF of $h$ is derived as

$$
f_{G}^{u g}(h)=f_{G}^{u g}(h>0)+f_{G}^{u g}(h=0) \delta(h),
$$


TABLE I

System Parameters USED Throughout Simulations

\begin{tabular}{lc}
\hline \hline Parameter & Setting \\
\hline \hline Wavelength $\lambda$ & $1550 \mathrm{~nm}$ \\
Responsivity $R$ & 0.9 \\
Aperture radius $r_{a}$ & $10 \mathrm{~cm}$ \\
Optical bandwidth $B_{o}$ & $10 \mathrm{~nm}$ \\
Electrical bandwidth $B_{e}$ & $1 \mathrm{GHz}$ \\
Spectral radiance $N_{b}(\lambda)$ & $10^{-3} \mathrm{~W} / \mathrm{cm}^{2}$-m-srad \\
Scattering coefficient $\zeta$ & $1 \mathrm{Km}^{-1}$ \\
SD of UAV position $\sigma_{p}$ & $20 \mathrm{~cm}$ \\
SD of ground station position $\sigma_{g}$ & $20 \mathrm{~cm}$ \\
Target SNR $\Upsilon_{t h}$ & $10 \mathrm{~dB}$ \\
\hline \hline
\end{tabular}

where $f_{G}^{u g}(h=0)=f_{G}^{u g}(h=0)$ and $f_{G}^{u g}(h>0)$ is obtained as

$$
\begin{aligned}
& f_{G}^{u g}(h>0) \simeq \sum_{j=0}^{J^{\prime}} \int_{0}^{\theta_{F O V}} \frac{\theta_{x y}}{\sigma_{\theta}^{2}} \exp \left(-\theta_{x y}^{2} / 2 \sigma_{\theta}^{2}\right) \\
& \quad \times\left(\mathcal{A}_{j}^{g u}(\alpha, \beta) \exp \left(-a_{j}^{\prime} \theta_{x y}^{2}\right) h^{\beta-1+j}\right. \\
& \left.\quad-\mathcal{A}_{j}^{g u}(\beta, \alpha) \exp \left(-b_{j}^{\prime} \theta_{x y}^{2}\right) h^{\alpha-1+j}\right) d \theta_{x y} \\
& =\sum_{j=0}^{J^{\prime}}\left(\frac{\mathcal{A}_{j}^{g u}(\alpha, \beta)}{1+2 a_{j}^{\prime} \sigma_{\theta}^{2}}\left(1-e^{-\left(a_{j}^{\prime}+1 / 2 \sigma_{\theta}^{2}\right) \theta_{F O V}^{2}}\right) h^{\beta-1+j}\right. \\
& \left.\quad-\frac{\mathcal{A}_{j}^{g u}(\beta, \alpha)}{1+2 b_{j}^{\prime} \sigma_{\theta}^{2}}\left(1-e^{-\left(b_{j}^{\prime}+1 / 2 \sigma_{\theta}^{2}\right) \theta_{F O V}^{2}}\right) h^{\alpha-1+j}\right),
\end{aligned}
$$

where $a_{j}^{\prime}=\frac{Z^{2}}{2 \sigma_{p g}^{2}}+\frac{Z^{2} \gamma_{g u}^{2}}{2 \sigma_{p g}^{2}\left(\beta-\gamma_{g u}^{2}+j\right)}$ and $b_{j}^{\prime}=\frac{Z^{2}}{2 \sigma_{p g}^{2}}+$ $\frac{Z^{2} \gamma_{g u}^{2}}{2 \sigma_{p g}^{2}\left(\alpha-\gamma_{g u}^{2}+j\right)}$. For low values of $h,(40)$ can be simplified

$$
\begin{aligned}
f_{G}^{u g}(h>0) & \simeq \frac{\pi \gamma_{g u}^{2}\left(\frac{\alpha \beta}{A_{0} h_{l}}\right)^{\beta} \sin ^{-1}((\alpha-\beta) \pi) h^{\beta-1}}{\Gamma(\alpha) \Gamma(\beta) \Gamma(-(\alpha-\beta)+1)\left|-\left(\beta-\gamma_{g u}^{2}\right)\right|} \\
& \times \frac{1}{1+2 a_{0}^{\prime} \sigma_{\theta}^{2}}\left(1-e^{-\left(a_{0}^{\prime}+1 / 2 \sigma_{\theta}^{2}\right) \theta_{F O V}^{2}}\right) .
\end{aligned}
$$

The PDF of $h$ for UG link under LN turbulence is given in Appendix B. The case of negligible turbulence is also considered in Appendix C.

Notice that the proposed channel models were initially characterized by nine RVs, which makes analytical calculation of the link performance very cumbersome. The proposed closed-form expressions for UU, GU, and UG links allow a significant reduction of the computational complexity.

\section{NumeriCAl Results AND Discussion}

Here, we firstly use classical Monte-Carlo simulations in order to verify the accuracy of the proposed expressions. We also investigate the impact of different Tx and Rx parameters and link range on the performance of the three considered links. The system parameters we use for performance evaluation are provided in Table I (SD denotes standard deviation). For the analytical results, we set $M=64$.

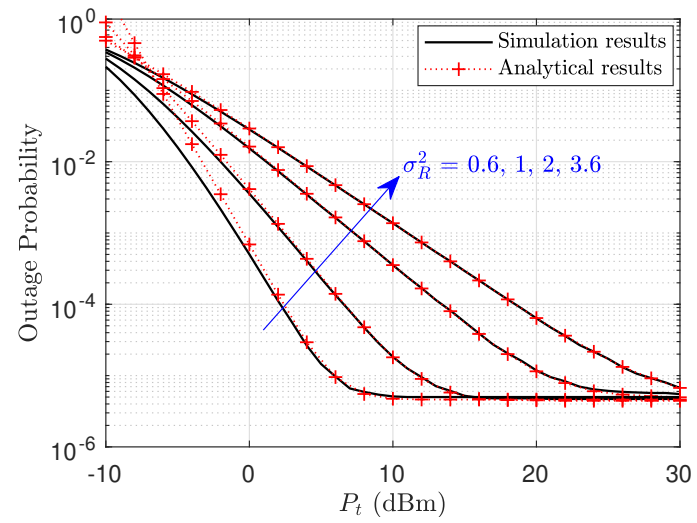

Fig. 4. Outage Probability of the UU link versus $P_{t}$ over GG turbulence model for $\theta_{F O V}=7 \mathrm{mrad}, \sigma_{\theta}=1 \mathrm{mrad}, w_{z}=2 \mathrm{~m}, Z=250 \mathrm{~m}$ and different values of $\sigma_{R}^{2}$.

Let us first investigate the performance of the considered system under different turbulence conditions. In Fig. 4, we have depicted $\mathcal{P}_{\text {out }}$ of the UU link over GG turbulence for different values of $\sigma_{R}^{2}$. We firstly notice that, at low values of $P_{t}$, as expected, the effect of turbulence on $\mathcal{P}_{\text {out }}$ is very significant. More importantly, regardless of turbulence strength, $\mathcal{P}_{\text {out }}$ is floored at high values of $P_{t}$. Indeed, according to (4), $h_{t h}$ approaches zero at large $P_{t}$. Then, substituting (24) in (3), we have $\mathcal{P}_{\text {out }} \simeq f_{h}^{u u}(h>0)=\sum_{m=0}^{M} \frac{\mathcal{H}(m) m !}{2}$. According to (46), $\mathcal{H}(m)$ only depends on $\theta_{F O V}$ and $\sigma_{\theta}^{2}$, and hence, $\mathcal{P}_{\text {out }}$ does not depend on $P_{t}$ nor on the turbulence strength. In addition, we notice a perfect match between the analytical and simulation-based results for moderate to strong range of atmospheric turbulence and relatively low $\mathcal{P}_{\text {out }}$ values.

For weak turbulence conditions and relatively high $\mathcal{P}_{\text {out }}$, there is a gap between the analytical and simulation results, which is in fact due to the approximations made in (17). More specifically, according to (7), $f_{G}\left(h_{a}\right)$ is a function of $k_{\varepsilon}(\xi)$, where $\varepsilon=\alpha-\beta$ and $\xi=2 \sqrt{\alpha \beta h_{a}}$. For conventional FSO links, we have typically $4>\sigma_{R}^{2}>0.05$ corresponding to $3>\varepsilon>1.5$. For this range of $\varepsilon$, (17) is valid approximately for $\xi<18$. For smaller values of $\sigma_{R}^{2}$ (weak turbulence), parameters $\alpha$ and $\beta$ are larger, giving rise to larger $h_{a}$, and hence, larger $\xi$, which compromises the validity of (17).To resolve this problem, we resort to the $\mathrm{LN}$ turbulence model for the weak turbulence regime, where our approximations (presented in Appendix B) remain more accurate. For instance, in Figs. 5a-5c, we have presented plots of $\mathcal{P}_{\text {out }}$ as a function of $P_{t}$ for different values of $\theta_{F O V}$ for $\mathrm{UU}, \mathrm{UG}$, and GU links for the cases of $\mathrm{LN}$ turbulence with $\sigma_{\operatorname{Ln} h_{a}}^{2}=0.1$ and negligible turbulence. Several important observations can be made from Fig. 5. Firstly, as expected, the UU link has its worst performance in terms of $\mathcal{P}_{\text {out }}$, compared with UG and GU links. Notice that the Rx orientation deviations affect $h_{p a}$ whereas those of Tx affect both $h_{p g}$ and $h_{p a}$. Also, remember that we have neglected $\mathrm{Tx} / \mathrm{Rx}$ orientation deviations when they are mounted on a ground station. Consequently, the GU link has a better performance than the UG link. Secondly, at relatively low $P_{t}$, smaller values of $\theta_{F O V}$ result in a slightly improved performance, which is due to reduced background 


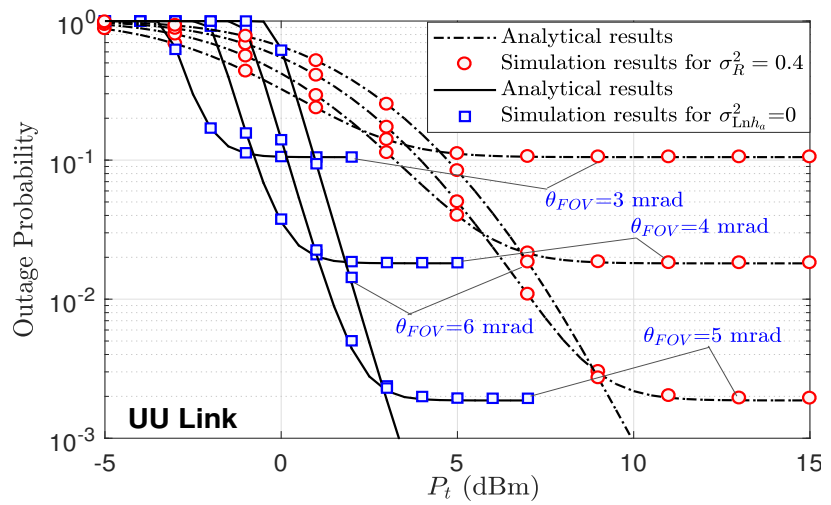

(a)

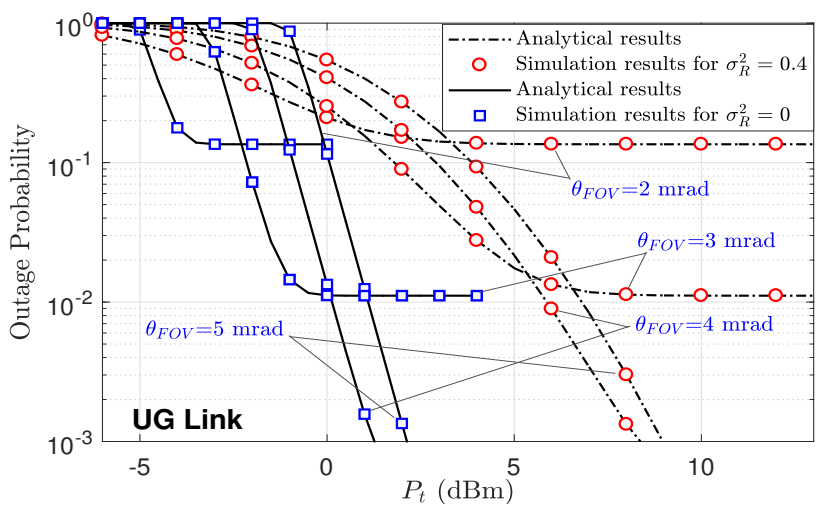

(b)

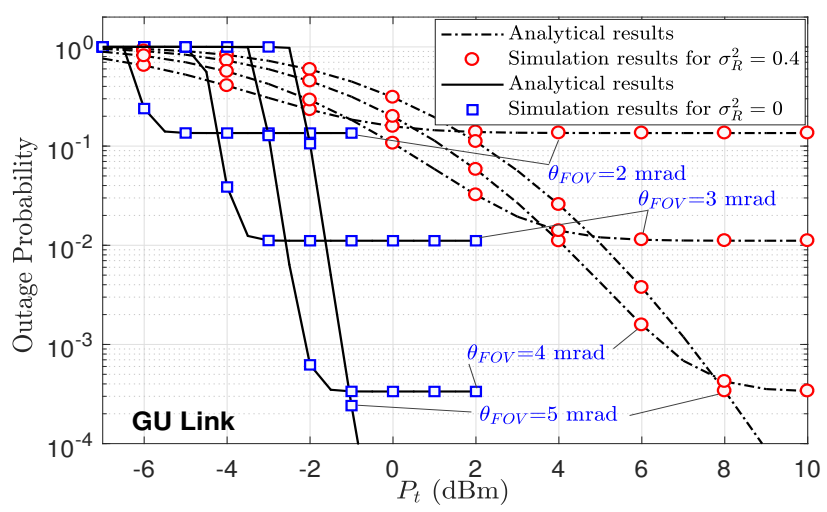

(c)

Fig. 5. Outage probability versus $P_{t}$ over $\mathrm{LN}$ turbulence model for different values of $\theta_{F O V} \cdot w_{z}=3.5 \mathrm{~m}, \sigma_{\theta}=1 \mathrm{mrad}, Z=500 \mathrm{~m}$ and for a) $\mathrm{UU}, \mathrm{b}$ ) UG and c) GU links.

noise level at the Rx. However, for relatively small $\theta_{F O V}$, increased $P_{t}$ results in a $\mathcal{P}_{\text {out }}$ floor. Indeed, as we see from the derivations of Section III, in such situations, $\mathcal{P}_{\text {out }}$ just depends on the AOA fluctuations and $\theta_{F O V}$. As we can notice from (49), (26) and (46) for UU link, increasing $\theta_{F O V}$ allows a decrease in $\mathcal{P}_{\text {out }}$ due to AOA fluctuations. Meanwhile, a larger $\theta_{F O V}$ results in an increased background noise level; thus the importance of finding an optimal value for $\theta_{F O V}$ to minimize $\mathcal{P}_{\text {out }}$ at a given $P_{t}$ (as we will discuss later). Meanwhile, we again notice the perfect match between simulation and analytical calculation results.
To get more insight into the importance of optimizing $w_{z}$ and $\theta_{F O V}$, we have presented for the UU link plots of $\mathcal{P}_{\text {out }}$ versus a wide range of beam waist and FOV in Figs. (6a) and (6b) for $P_{t}=15$ and $25 \mathrm{dBm}$, respectively. We notice the high sensitivity of the link performance to $w_{z}$ and $\theta_{F O V}$. Moreover, by increasing $P_{t}$ the optimum $\theta_{F O V}$ and $w_{z}$ are increased: they are $11.8 \mathrm{mrad}$ and $3.2 \mathrm{~m}$ for $P_{t}=15 \mathrm{dBm}$, compared to $16.5 \mathrm{mrad}$ and $3.8 \mathrm{~m}$ for $P_{t}=25 \mathrm{dBm}$. The dependence of the optimum values on $P_{t}$ suggests that they are also function of the background noise level in practice.

In addition to $P_{t}$, other channel parameters such as link range $Z$ and the variance of orientation fluctuations of Tx/Rx $\sigma_{\theta}^{2}$ affect the link performance and consequently, the optimum values of $\theta_{F O V}$ and $w_{z}$. This is shown in Tables II and III for $P_{t}=15 \mathrm{dBm}$ and different values of $Z$ and $\sigma_{\theta}$, respectively. Also, the minimum achievable $\mathcal{P}_{\text {out }}$ and the corresponding link interruption probability (due to the AOA fluctuations) are given. We notice from Table II that for a fixed $\sigma_{\theta}$, we need a larger $\theta_{F O V}$ at shorter $Z$ to minimize $\mathcal{P}_{\text {out }}$ which is due to a lower link interruption probability for a given $\sigma_{\theta}$. The optimum $\theta_{F O V}$ makes a compromise between increased geometric loss and the Rx background noise level on one hand, and the decrease in the link interruption probability on the other hand. However, the change in $\sigma_{\theta}$ and $Z$ (and hence, in $h_{l}$ ) makes it very difficult to predict the behavior of the optimum $w_{z}$. From Table III, we notice that as $\sigma_{\theta}$ decreases, the optimal $\theta_{F O V}$ and $w_{z}$ decrease. This is reasonable because for smaller pointing errors, we can make the beam more directional to reduce the geometric loss and the Rx background noise at the same time. Reasonably, we obtain a smaller $\mathcal{P}_{\text {out }}$ as well.

\section{Concluding Remarks and Future Directions}

The goal of this paper was to provide an accurate and computationally efficient channel model for the case of MR UAVbased FSO communications. Thanks to the proposed model, we also considered the optimization of the $\mathrm{Tx} / \mathrm{Rx}$ tunable parameters (such as beam waist and FOV) in order to achieve the best performance, e.g., the lowest outage probability. The optimal parameters highly depend on channel conditions such as range, turbulence strength, payload vibrations, transmit power, and background noise power. The proposed channel model, which takes into account as many as nine random parameters, was shown to be of significantly high accuracy, allowing easy calculation and tuning of optimal Tx/Rx parameters in an adaptive FSO system, thus removing the need to complex and time consuming Monte Carlo simulations.

Lastly, as a future research direction, one can consider deriving closed-form expressions for the tunable parameters (i.e., the Tx beam waist and the $\mathrm{Rx}$ FOV). Another direction concerns the more general case of mobile UAVs, whose instantaneous positions are calculated through accurate information exchange between them on speed, acceleration, distance, and movement direction. There, the proposed channel model should be completed by taking into account estimation errors on such parameters. 


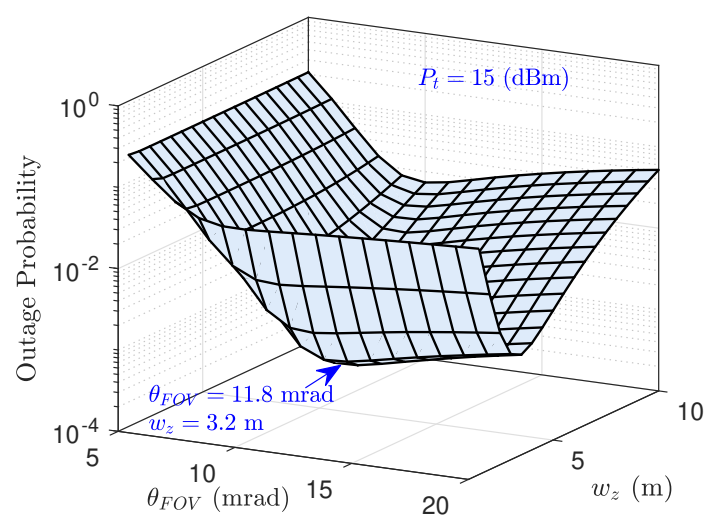

(a)

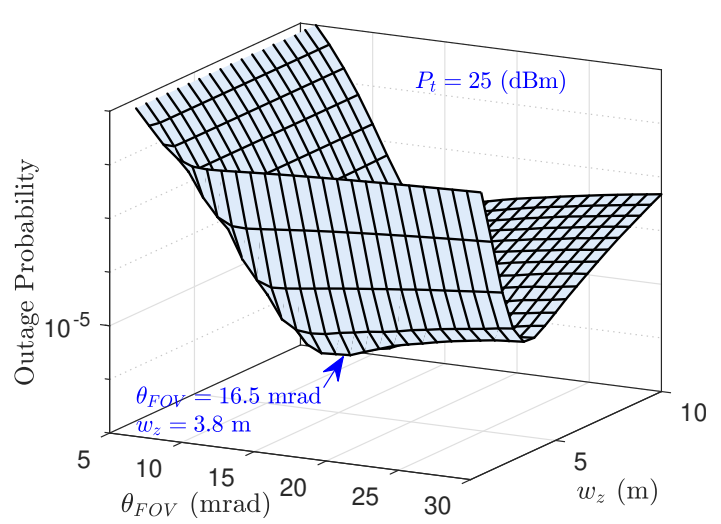

(b)

Fig. 6. Outage probability of UU link versus $\theta_{F O V}$ and $w_{z}$ over GG turbulence model for $Z=500 \mathrm{~m}, \sigma_{R}^{2}=1$ and a) $P_{t}=15 \mathrm{dBm}$ and b) $P_{t}=25 \mathrm{dBm}$.

TABLE II

OPtIMUM VALUES OF $\theta_{F O V}$ AND $w_{z}$ TO ACHIEVE MinimUM OUTAGE PROBABILITY OVER GG TURBULENCE MODEL FOR $\sigma_{R}^{2}=1, \sigma_{\theta}=2$ mrad AND $P_{t}=15 \mathrm{dBm}$ AND DIFFERENT VALUES OF $Z$.

\begin{tabular}{|c|c|c|c|c|c|c|c|c|c|c|c|c|}
\hline & \multicolumn{4}{|c|}{ UAV-to-UAV } & \multicolumn{4}{|c|}{ UAV-to-Ground } & \multicolumn{4}{|c|}{ Ground-to-UAV } \\
\hline $\begin{array}{c}Z \\
(\mathrm{~km})\end{array}$ & $\begin{array}{l}\theta_{F O V} \\
(\mathrm{mrad})\end{array}$ & $\begin{array}{l}w_{z} \\
(\mathrm{~m})\end{array}$ & $\mathcal{P}_{\text {out }}$ & $f_{h}^{u u}(h=0)$ & $\begin{array}{l}\theta_{F O V} \\
(\mathrm{mrad})\end{array}$ & $\begin{array}{c}w_{z} \\
(\mathrm{~m})\end{array}$ & $\mathcal{P}_{\text {out }}$ & $f_{h}^{u g}(h=0)$ & $\begin{array}{l}\theta_{F O V} \\
(\mathrm{mrad})\end{array}$ & $\begin{array}{l}w_{z} \\
(\mathrm{~m})\end{array}$ & $\mathcal{P}_{\text {out }}$ & $f_{h}^{g u}(h=0)$ \\
\hline 0.25 & 14.3 & 2.1 & $1.9 \times 10^{-5}$ & $1.7 \times 10^{-6}$ & 10.7 & 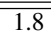 & $3.8 \times 10^{-6}$ & $5.3 \times 10^{-7}$ & 15.4 & 0.6 & $6.2 \times 10^{-8}$ & $1.1 \times 10^{-9}$ \\
\hline 0.5 & 12.4 & 3.6 & $6.4 \times 10^{-4}$ & $5.7 \times 10^{-5}$ & 9.6 & 3.2 & $1.4 \times 10^{-4}$ & $1.3 \times 10^{-5}$ & 14.8 & 0.6 & $9.4 \times 10^{-8}$ & $2.3 \times 10^{-9}$ \\
\hline 1 & 9.9 & 5.9 & 0.11 & $2.2 \times 10^{-2}$ & 7.3 & 5.4 & $6.3 \times 10^{-2}$ & $1.4 \times 10^{-2}$ & 13.8 & 0.5 & $1.8 \times 10^{-7}$ & $2.6 \times 10^{-8}$ \\
\hline 1.5 & 7.8 & 7.7 & 0.32 & 0.1 & 5.8 & 6.6 & 0.12 & $8.2 \times 10^{-2}$ & 12.7 & 0.5 & $7.3 \times 10^{-7}$ & $8.9 \times 10^{-8}$ \\
\hline
\end{tabular}

TABLE III

Optimum VAlUES of $\theta_{F O V}$ AND $w_{z}$ TO ACHIEVE MINIMUM OUTAGE PROBABILITY OVER GG TURBULENCE MODEL FOR $\sigma_{R}^{2}=1, Z=500$ M AND $P_{t}=15 \mathrm{dBm}$ AND DIFFERENT VALUES OF $\sigma_{\theta}$.

\begin{tabular}{|c|c|c|c|c|c|c|c|c|c|c|c|c|}
\hline & \multicolumn{4}{|c|}{ UAV-to-UAV } & \multicolumn{4}{|c|}{ UAV-to-Ground } & \multicolumn{4}{|c|}{ Ground-to-UAV } \\
\hline $\begin{array}{c}\sigma_{\theta} \\
(\mathrm{mrad})\end{array}$ & $\begin{array}{c}\theta_{F O V} \\
(\mathrm{mrad})\end{array}$ & $\begin{array}{l}w_{z} \\
(\mathrm{~m}) \\
\end{array}$ & $\mathcal{P}_{\text {out }}$ & $f_{h}^{u u}(h=0)$ & $\begin{array}{c}\theta_{F O V} \\
(\mathrm{mrad})\end{array}$ & $\begin{array}{l}w_{z} \\
(\mathrm{~m})\end{array}$ & $\mathcal{P}_{\text {out }}$ & $f_{h}^{u g}(h=0)$ & $\begin{array}{c}\theta_{F O V} \\
(\mathrm{mrad})\end{array}$ & $\begin{array}{l}w_{z} \\
(\mathrm{~m})\end{array}$ & $\mathcal{P}_{\text {out }}$ & $f_{h}^{y u}(h=0)$ \\
\hline$\overline{88}$ & 29.1 & $\overline{5.2}$ & 0.16 & $\overline{3.4 \times 10^{-2}}$ & 23.4 & 3.3 & $\overline{5.3 \times 10^{-2}}$ & $\overline{1.1 \times 10^{-2}}$ & 36,2 & 1.3 & $2.6 \times 10^{-4}$ & $\overline{6.5 \times 10^{-5}}$ \\
\hline 4 & 21.1 & 3.3 & $8.3 \times 10^{-3}$ & $1.1 \times 10^{-3}$ & 16.4 & 2.8 & $1.9 \times 10^{-3}$ & $5.4 \times 10^{-4}$ & 19.1 & 1.4 & $6.5 \times 10^{-5}$ & $1.1 \times 10^{-5}$ \\
\hline 2 & 13.2 & 2.1 & $1.7 \times 10^{-4}$ & $2.3 \times 10^{-5}$ & 9.9 & 1.74 & $3.6 \times 10^{-5}$ & $6.6 \times 10^{-6}$ & 10.1 & 1.6 & $1.6 \times 10^{-5}$ & $3.6 \times 10^{-6}$ \\
\hline 1 & 7.4 & 1.1 & $4.3 \times 10^{-6}$ & $1.5 \times 10^{-6}$ & 5.5 & 1.4 & $3.2 \times 10^{-6}$ & $3.4 \times 10^{-7}$ & 5.6 & 1.7 & $8.8 \times 10^{-7}$ & $2.1 \times 10^{-7}$ \\
\hline
\end{tabular}

APPENDIX A

DERIVATION OF EQ. (21)

According to Eq. (19), the RV $\theta_{a}$ conditioned on $\theta_{t x}$ and $\theta_{t y}$ has a Rician distribution expressed as

$$
f_{\theta_{a} \mid \theta_{x y}}\left(\theta_{a}\right)=\frac{\theta_{a}}{\sigma_{\theta}^{2}} \exp \left(-\frac{\theta_{a}^{2}+\theta_{x y}^{2}}{2 \sigma_{\theta}^{2}}\right) I_{0}\left(\frac{\theta_{a} \theta_{x y}}{\sigma_{\theta}^{2}}\right) .
$$

From (42) and (20), the distribution of $h_{p a}$ can be derived as

$$
\begin{aligned}
f_{h_{p a} \mid \theta_{x y}}\left(h_{p a}\right)= & F_{\theta_{a} \mid \theta_{x y}}\left(\theta_{F O V}\right) \delta\left(h_{p a}-1\right) \\
& +\left(1-F_{\theta_{a} \mid \theta_{x y}}\left(\theta_{F O V}\right)\right) \delta\left(h_{p a}\right),
\end{aligned}
$$

where

$$
F_{\theta_{a} \mid \theta_{x y}}\left(\theta_{F O V}\right)=\int_{0}^{\theta_{F O V}} f_{\theta_{a} \mid \theta_{x y}}\left(\theta_{a}\right) d \theta_{a} .
$$

Using the polynomial approximation for $I_{k}(x)$ from [28, Eq. (19)] and [29, Eq. (6) ], a closed-form expression for (44) can be derived as

$$
F_{\theta_{a} \mid \theta_{x y}}\left(\theta_{F O V}\right) \simeq 1-\exp \left(-\frac{\theta_{x y}^{2}}{2 \sigma_{\theta}^{2}}\right) \sum_{m=0}^{M} \mathcal{H}(m)\left(\frac{\theta_{x y}^{2}}{\sigma_{\theta}^{2}}\right)^{m},
$$

where

$$
\mathcal{H}(m)=\frac{2^{-m} M^{(1-2 m)} \Gamma(M+m) \Gamma\left(m+1, \frac{\theta_{F O V}^{2}}{2 \sigma_{\theta}^{2}}\right)}{(\Gamma(m+1))^{2} \Gamma(M-m+1)},
$$

and $\Gamma(.,$.$) denotes upper incomplete gamma function [27].$ Note that, when $M \rightarrow \infty$, Equation (45) degenerates into (44) [27]. Since $h=h_{p a} h^{\prime}$ and the RVs $h_{p a}$ and $h^{\prime}$ are independent conditioned on $\theta_{x y}$, the distribution of $\mathrm{RV} h$ conditioned on $\theta_{x y}$ can be obtained as

$$
\begin{aligned}
f_{h \mid \theta_{x y}}(h)= & \int_{0}^{\infty} f_{h \mid h^{\prime}, \theta_{x y}}(h) f_{h^{\prime} \mid \theta_{x y}}\left(h^{\prime}\right) d h^{\prime} \\
= & \int_{0}^{\infty} \frac{1}{h^{\prime}} f_{h_{p a} \mid \theta_{x y}}\left(\frac{h}{h^{\prime}}\right) f_{h^{\prime} \mid \theta_{x y}}\left(h^{\prime}\right) d h^{\prime} \\
= & \int_{0}^{\infty} \frac{f_{h^{\prime} \mid \theta_{x y}}\left(h^{\prime}\right)}{h^{\prime}}\left[F_{\theta_{a} \mid \theta_{x y}}\left(\theta_{F O V}\right) \delta\left(\frac{h-h^{\prime}}{h^{\prime}}\right)\right. \\
& \left.+\left(1-F_{\theta_{a} \mid \theta_{x y}}\left(\theta_{F O V}\right)\right) \delta\left(\frac{h}{h^{\prime}}\right)\right] d h^{\prime}
\end{aligned}
$$




$$
\begin{aligned}
f_{L}^{u u}(h>0)= & C_{a} h^{\gamma_{u u}^{2}-1} \int_{0}^{\infty} \frac{\theta_{x y}}{\sigma_{\theta}^{2}}\left(1-\exp \left(-\theta_{x y}^{2} / 2 \sigma_{\theta}^{2}\right) \sum_{m=0}^{M} \mathcal{H}(m)\left(\theta_{x y}^{2} / \sigma_{\theta}^{2}\right)^{m}\right) \\
& \times \exp \left[\left(Z^{2} / 2 \sigma_{p}^{2}-1 / 2 \sigma_{\theta}^{2}\right) \theta_{x y}^{2}\right] Q\left(\left(w_{z_{e q}}^{2} \ln \left(h / A_{0} h_{l}\right)+6 Z^{2} \theta_{x y}^{2}+C_{b}\right) / \sqrt{32 \sigma_{p}^{2} Z^{2} \theta_{x y}^{2}+C_{c}}\right) d \theta_{x y} .
\end{aligned}
$$

$$
\begin{aligned}
= & \int_{0}^{\infty} f_{h^{\prime} \mid \theta_{x y}}\left(h^{\prime}\right)\left[F_{\theta_{a} \mid \theta_{x y}}\left(\theta_{F O V}\right) \delta\left(h-h^{\prime}\right)\right. \\
& \left.+\left(1-F_{\theta_{a} \mid \theta_{x y}}\left(\theta_{F O V}\right)\right) \delta(h)\right] d h^{\prime} \\
= & F_{\theta_{a} \mid \theta_{x y}}\left(\theta_{F O V}\right) f_{h^{\prime} \mid \theta_{x y}}(h) \\
& +\left(1-F_{\theta_{a} \mid \theta_{x y}}\left(\theta_{F O V}\right)\right) \delta(h) .
\end{aligned}
$$

\section{APPENDIX B}

\section{DERIVATIONS OF CHANNEL Distribution OVER} LoG-Normal Turbulence Model

1) UAV-to-UAV Channel Modeling: Substituting (6), (13) and (14) in (15) and using [4, Eqs. (9), (10)], the distribution of $h^{\prime}=h_{l} h_{a} h_{p g}$ conditioned on $\theta_{x y}$ is obtained as

$$
\begin{aligned}
f_{h^{\prime} \mid \theta_{x y}}^{\mathrm{L}}\left(h^{\prime}\right) & =C_{a} h^{\prime \gamma_{u u}^{2}-1} \exp \left(Z^{2} \theta_{x y}^{2} / 2 \sigma_{p}^{2}\right) \\
\times & \times\left(\frac{w_{z_{e q}}^{2} \ln \left(h^{\prime} / A_{0} h_{l}\right)+6 Z^{2} \theta_{x y}^{2}+C_{b}}{\sqrt{32 \sigma_{p}^{2} Z^{2} \theta_{x y}^{2}+C_{c}}}\right),
\end{aligned}
$$

where $C_{a}=\gamma_{u u}^{2} /\left(A_{0} h_{l}\right)^{\gamma_{u u}^{2}} \times \exp \left(2 \sigma_{L n h_{a}}^{2} \gamma_{u u}^{2}\left(1+\gamma_{u u}^{2}\right)\right)$, $C_{b}=2 \sigma_{L n h_{a}}^{2} w_{z_{e q}}^{2}\left(1+2 \gamma_{u u}^{2}\right), C_{c}=4 \sigma_{L n h_{a}}^{2} w_{z_{e q}}^{4}$ and $Q($.$) is$ the well-known $Q$-function. Substituting (13), (48), (21) and (22) in (23), we obtain the PDF of $h$ for UU link as

$$
f_{L}^{u u}(h)=f_{L}^{u u}(h>0)+f_{L}^{u u}(h=0) \delta(h),
$$

where $f_{L}^{u u}(h=0)=f_{G}^{u u}(h=0)$ and $f_{L}^{u u}(h>0)$ is expressed in (50).

2) Ground-to-UAV Channel Modeling: Using (29) and [18, Eqs, (10), (11), (14)], the distribution of $h^{\prime}$ for the GU link is obtained as

$$
f_{h^{\prime}}^{\mathrm{L}},\left(h^{\prime}\right)=C_{e} h^{\prime\left(\gamma_{g u}^{2}-1\right)} Q\left(\frac{\ln \left(h^{\prime} / A_{0} h_{l}\right)+C_{d}}{2 \sigma_{\operatorname{Ln} h_{a}}}\right),
$$

where $C_{e}=\frac{\gamma_{g u}^{2}}{\left(A_{0} h_{l}\right)^{\gamma_{g u}^{2}}} \exp \left(2 \sigma_{\operatorname{Ln} h_{a}}^{2} \gamma_{g u}^{2}\left(1+\gamma_{g u}^{2}\right)\right)$, and $C_{d}=$ $2 \sigma_{\operatorname{Lnh} h_{a}}^{2}\left(1+2 \gamma_{g u}^{2}\right)$. From (21), (51) and (33), the distribution of $h$ for GU link can be obtained as

$$
f_{L}^{g u}(h)=f_{L}^{g u}(h>0)+f_{L}^{g u}(h=0) \delta(h),
$$

where $f_{L}^{g u}(h=0)=f_{G}^{g u}(h=0)$ and $f_{L}^{g u}(h>0)$ is obtained as

$$
\begin{aligned}
f_{L}^{g u}(h>0) & =C_{e}\left[1-\exp \left(-\theta_{F O V}^{2} / 2 \sigma_{\theta}^{2}\right)\right] h^{\left(\gamma_{g u}^{2}-1\right)} \\
& \times Q\left(\left(\ln \left(\frac{h}{A_{0} h_{l}}\right)+C_{d}\right) / 2 \sigma_{\operatorname{Ln} h_{a}}\right) .
\end{aligned}
$$

3) UAV-to-Ground Channel Modeling: Using (13), (48) and (38), the PDF of $h$ for UG link is obtained as

$$
f_{L}^{u g}(h)=f_{L}^{u g}(h>0)+f_{L}^{u g}(h=0) \delta(h),
$$

where $f_{L}^{u g}(h=0)=f_{L}^{g u}(h=0)$ and $f_{L}^{u g}(h>0)$ is given in (55).

\section{APPENDIX C}

DeRivations of CHANNEl Distribution IN THE

\section{ABSENCE OF ATMOSPHERIC TURbUlEnCE}

Here, we consider the special case where the atmospheric turbulence has a negligible effect, i.e., $h^{\prime}=h_{l} h_{p g}$.

1) For UU Link: Under such condition, distribution of $h$ conditioned on $\theta_{x y}$ can be simplified as

$$
f_{h^{\prime} \mid \theta_{x y}}(h)=\frac{1}{h_{l}} f_{h_{p g} \mid \theta_{x y}}\left(h^{\prime} / h_{l}\right), \text { for } 0 \leq h^{\prime} \leq A_{0} h_{l} .
$$

Now, by substituting (56) in (22), we have

$$
\begin{aligned}
& f_{h \mid \theta_{x y}}(h)=\frac{1}{h_{l}} F_{\theta_{a} \mid \theta_{x y}}\left(\theta_{F O V}\right) f_{h_{p g} \mid \theta_{x y}}\left(h / h_{l}\right) \\
& +\left(1-F_{\theta_{a} \mid \theta_{x y}}\left(\theta_{F O V}\right)\right) \delta(h), \text { for } 0 \leq h \leq A_{0} h_{l} .
\end{aligned}
$$

According to (23) and (57) and also using [27, Eq. (8.445)] and [27, Eq. (3.381.4)], after some algebra, (49) is simplified as

$$
\begin{aligned}
f_{h}^{u u, l}(h)= & \left(\frac{h}{A_{0} h_{l}}\right)^{\gamma^{2}-1} \sum_{k=0}^{\infty} F(k)\left(\ln \left(\frac{h}{A_{0} h_{l}}\right)\right)^{k} \\
& +\sum_{m=0}^{M} \frac{\mathcal{H}(m) m !}{2}, \quad \text { for } \quad 0 \leq h \leq A_{0} h_{l},
\end{aligned}
$$

where, for a given $k, F(k)$ is a constant and is obtained as

$$
\begin{aligned}
F(k)= & \frac{\gamma^{2}}{2 h_{l} A_{0} \sigma_{\theta}^{2}} \frac{1}{(k !)^{2}}\left(-\frac{Z^{2} \gamma^{2}}{4 \sigma_{p}^{2}}\right)^{k}\left\{\frac{\Gamma(k+1)}{\left(\frac{Z^{2}}{4 \sigma_{p}^{2}}+\frac{1}{2 \sigma_{\theta}^{2}}\right)^{k+1}}\right. \\
& \left.-\sum_{m=0}^{M} \frac{\mathcal{H}(m) \Gamma(k+m+1)}{\left(\frac{Z^{2}}{4 \sigma_{p}^{2}}+\frac{1}{\sigma_{\theta}^{2}}\right)^{k+1}\left(\frac{Z^{2} \sigma_{\theta}^{2}}{4 \sigma_{p}^{2}}+1\right)^{m}}\right\}
\end{aligned}
$$

2) For UG Link: At this condition, the distribution of $\mathrm{RV} h^{\prime}$ conditioned on $\theta_{x y}$ can be obtained as (57) and the distribution of $h$ for UG link can be simplified as

$$
\begin{aligned}
& f_{h}^{u g, l}(h)=\int_{0}^{\theta_{F O V}} \frac{\gamma_{g u}^{2}}{h_{l} A_{0}} \exp \left(-\frac{\theta_{x y}^{2} Z^{2}}{2 \sigma_{p g}^{2}}\right)\left(\frac{h}{h_{l} A_{0}}\right)^{\gamma_{g u}^{2}-1} \\
& \times I_{0}\left(Z \theta_{x y} \sqrt{-\frac{2 \gamma_{g u}^{2}}{\sigma_{p g}^{2}} \ln \left(\frac{h}{h_{l} A_{0}}\right)}\right) \frac{\theta_{x y}}{\sigma_{\theta}^{2}} \exp \left(-\frac{\theta_{x y}^{2}}{2 \sigma_{\theta}^{2}}\right) d \theta_{x y} \\
& +\exp \left(-\theta_{F O V}^{2} / 2 \sigma_{\theta}^{2}\right) \delta(h), \quad \text { for } \quad 0 \leq h \leq A_{0} h_{l} .
\end{aligned}
$$

According to [27, Eq. (8.445)] and [27, Eq. (3.381.2)] and after some manipulation, we have

$$
\begin{gathered}
f_{h}^{u g, l}(h)=\left(\frac{h}{h_{l} A_{0}}\right)^{\gamma_{g u}^{2}-1} \sum_{k=1}^{\infty} F_{u g}(k)\left(-\ln \left(\frac{h}{h_{l} A_{0}}\right)\right)^{k} \\
+\exp \left(-\theta_{F O V}^{2} / 2 \sigma_{\theta}^{2}\right) \delta(h), \quad \text { for } \quad 0 \leq h \leq A_{0} h_{l} .
\end{gathered}
$$




$$
f_{L}^{u g}(h>0)=C_{a} h^{\gamma_{g u}^{2}-1} \int_{0}^{\theta_{F O V}} \frac{\theta_{x y}}{\sigma_{\theta}^{2}} \exp \left[\left(\frac{Z^{2}}{\sigma_{p g}^{2}}-\frac{1}{2 \sigma_{\theta}^{2}}\right) \theta_{x y}^{2}\right] Q\left(\frac{w_{z_{e q}}^{2} \ln \left(h / A_{0} h_{l}\right)+6 Z^{2} \theta_{x y}^{2}+C_{b}}{\sqrt{16 \sigma_{p g}^{2} Z^{2} \theta_{x y}^{2}+C_{c}}}\right) d \theta_{x y} .
$$

where, for a given $k, F_{u g}(k)$ is a constant and is obtained as

$$
\begin{aligned}
F_{u g}(k) & =\frac{\gamma_{g u}^{2}}{h_{l} A_{0} \sigma_{\theta}^{2}}\left(Z^{2} \gamma_{g u}^{2} / 2 \sigma_{p g}^{2}\right)^{k} \\
& \times \sum_{n=1}^{\infty} \frac{\left(\theta_{F O V}\right)^{n+k+1}}{n !(k+n+1)}\left[-\left(\frac{Z^{2}}{2 \sigma_{p g}^{2}}+\frac{1}{2 \sigma_{\theta}^{2}}\right)\right]^{n} .
\end{aligned}
$$

3) For GU Link: Similar to our previous derivations, the distribution of $h$ in the GU link can be expressed as

$$
\begin{aligned}
f_{h}^{g u, l}= & {\left[1-\exp \left(-\theta_{F O V}^{2} / 2 \sigma_{\theta}^{2}\right)\right]\left(\gamma_{g u}^{2} / A_{0}^{\gamma_{g u}^{2}}\right) h^{\gamma_{g u}^{2}-1} } \\
& +\exp \left(-\theta_{F O V}^{2} / 2 \sigma_{\theta}^{2}\right) \delta(h) .
\end{aligned}
$$

\section{REFERENCES}

[1] M. A. Khalighi and M. Uysal, "Survey on free space optical communication: A communication theory perspective," IEEE Commun. Surv. Tutorials, vol. 16, no. 4, pp. 2231-2258, 2014.

[2] Z. Ghassemlooy, W. Popoola, and S. Rajbhandari, Optical wireless communications. CRC Press Boca Raton, FL, 2012.

[3] D. Schulz, V. Jungnickel, C. Alexakis, M. Schlosser, J. Hilt, A. Paraskevopoulos, L. Grobe, P. Farkas, and R. Freund, "Robust optical wireless link for the backhaul and fronthaul of small radio cells," $J$. Lightw. Technol., vol. 34, no. 6, pp. 1523-1532, 2016.

[4] F. Yang, J. Cheng, and T. A. Tsiftsis, "Free-space optical communication with nonzero boresight pointing errors," IEEE Trans. Commun., vol. 62, no. 2, pp. 713-725, 2014.

[5] B. Zhu, J. Cheng, M.-S. Alouini, and L. Wu, "Relay placement for FSO multihop DF systems with link obstacles and infeasible regions," IEEE Trans. Wireless Commun., vol. 14, no. 9, pp. 5240-5250, 2015.

[6] L. Li et al., "80-Gbit/s 100-m free-space optical data transmission link via a flying UAV using multiplexing of orbital-angular-momentum beams," arXiv preprint arXiv:1708.02923, 2017.

[7] G. A. Cap, H. H. Refei, and J. J. Sluss, "Optical tracking and autoalignment transceiver system," IEEE Aerosp. Electron. Syst. Mag., vol. 25, no. 9, pp. 26-34, 2010.

[8] B. Moision et al., "Demonstration of free-space optical communication for long-range data links between balloons on Project Loon," in Proc. of SPIE Vol, vol. 10096, 2017, pp. 100960Z-1.

[9] C. Chen et al., "High-speed optical links for UAV applications," vol. 10096, pp. $1009615-1,2017$.

[10] H. Kaushal and G. Kaddoum, "Optical communication in space: Challenges and mitigation techniques," IEEE Commun. Surveys Tuts., vol. 19, no. 1, pp. 57-96, 2017.

[11] E. Kalantari, M. Z. Shakir, H. Yanikomeroglu, and A. Yongacoglu, "Backhaul-aware robust 3D drone placement in 5G+ wireless networks," in 2017 IEEE Int. Conf. Commun. (ICC),. IEEE, 2017, pp. 109-114.
[12] A. Kaadan, H. H. Refai, and P. G. LoPresti, "Multielement FSO transceivers alignment for inter-UAV communications," J. Lightw. Technol., vol. 32, no. 24, pp. 4183-4193, 2014.

[13] M. Alzenad, M. Z. Shakir, H. Yanikomeroglu, and M.-S. Alouini, "FSO-based vertical backhaul/fronthaul framework for $5 \mathrm{G}+$ wireless networks," IEEE Commun. Mag., vol. 56, no. 1, pp. 218-224, 2018.

[14] W. Fawaz, C. Abou-Rjeily, and C. Assi, "UAV-Aided Cooperation for FSO Communication Systems," IEEE Commun. Mag., vol. 56, no. 1, pp. 70-75, 2018.

[15] P. J. Cruz and R. Fierro, "Towards optical wireless communications between micro unmanned aerial and ground systems," pp. 669-676, 2015.

[16] M. Khan, M. Yuksel, and G. Winkelmaier, "GPS-free maintenance of a free-space-optical link between two autonomous mobiles," IEEE Trans. Mobile Comput., vol. 16, no. 6, pp. 1644-1657, 2017.

[17] A. Kaadan, H. Refai, and P. Lopresti, "Spherical FSO receivers for UAV communication: geometric coverage models," IEEE Trans. Aerosp. Electron. Syst., vol. 52, no. 5, pp. 2157-2167, 2016.

[18] A. A. Farid and S. Hranilovic, "Outage capacity optimization for freespace optical links with pointing errors," J. Light. Technol., vol. 25, no. 7, pp. 1702-1710, 2007.

[19] S. Huang and M. Safari, "Free-space optical communication impaired by angular fluctuations," IEEE Trans. Wireless Commun., 2017.

[20] M. A. Khalighi, F. Xu, Y. Jaafar, and S. Bourennane, "Double-laser differential signaling for reducing the effect of background radiation in free-space optical systems," IEEE/OSA J. Opt. Commun. Net., vol. 3, no. 2, pp. 145-154, Feb. 2011.

[21] F. Xu, M. A. Khalighi, and S. Bourennane, "Impact of different noise sources on the performance of PIN- and APD-based FSO receivers," IEEE ConTEL Conf., pp. 211-218, June 2011, Graz, Austria.

[22] M. T. Dabiri, S. M. S. Sadough, and M. A. Khalighi, "FSO channel estimation for OOK modulation with APD receiver over atmospheric turbulence and pointing errors," Opt. Commun., vol. 402, pp. 577-584, 2017.

[23] N. S. Kopeika and J. Bordogna, "Background noise in optical communication systems," Proceedings of the IEEE, vol. 58, no. 10, pp. 1571$1577,1970$.

[24] L. C. Andrews and R. L. Phillips, Laser beam propagation through random media. SPIE press Bellingham, WA, 2005, vol. 52.

[25] M.-A. Khalighi, N. Schwartz, N. Aitamer, and S. Bourennane, "Fading reduction by aperture averaging and spatial diversity in optical wireless systems," J. opt. commun. netw., vol. 1, no. 6, pp. 580-593, 2009.

[26] Wolfram, "The wolfram functions site: http://functions.wolfram.com/," 2001.

[27] I. S. Gradshteyn and I. M. Ryzhik, Table of integrals, series, and products. 7th ed. Academic press, 2007.

[28] L. Li, F. Li, and F. Gross, "A new polynomial approximation for J $\nu$ Bessel functions," Applied mathematics and computation, vol. 183, no. 2, pp. 1220-1225, 2006.

[29] P. C. Sofotasios and S. Freear, "Novel expressions for the Marcum and one dimensional Q-functions," pp. 736-740, 2010. 